\title{
Bem-estar subjetivo, regulação emocional e orientação motivacional em atletas de alta competição
}

\section{Subjective well-being, emotional regulation and motivational orientation of competition athletes}

\author{
Sílvia Amado Cordeiro, Priscila Martins, Cristina Costa-Lobo \\ Instituto de Desenvolvimento Humano Portucalense
}

\begin{abstract}
Resumo
No contexto desportivo, as respostas emocionais dos atletas e a sua capacidade para as regular, parece ser uma competência fundamental para o sucesso desportivo. Neste âmbito, estudaram-se 50 jovens atletas de um clube de futebol português, afim de se analisar a relação do bem-estar subjetivo, da regulação emocional e da orientação motivacional segundo o rendimento desportivo. Os resultados sugerem que a orientação motivacional parece estar relacionada com o alto rendimento no desporto. Apuraram-se ainda, diferenças na regulação emocional em função do rendimento. Discute-se acerca das implicações do bem-estar subjetivo, da regulação emocional e da orientação motivacional, no rendimento desportivo.

Palavras-chave: Rendimento desportivo, bem-estar subjetivo, regulação emocional, orientação motivacional.
\end{abstract}

\section{Abstract}

In the sporting context, athlete's emotional responses and their ability to regulate them seem to be a fundamental competence for sports success. In this context, 50 young athletes from a Portuguese soccer club were studied in order to analyse the relationship of subjective well-being, emotional regulation and motivational orientation according to sports performance. The results suggest that motivational orientation seems to be related to high performance in sports. Differences were also found in emotional regulation as a function of income. It discusses the implications of subjective well-being, emotional regulation, and motivational orientation on sports performance.

Keywords: Subjective well-being, emotional regulation, motivational orientation, sports performance

\section{Introdução}

O desempenho excelente no desporto tem vindo a suscitar o interesse da comunidade científica, em particular na área da psicologia e das ciências do desporto, originando um aumento da investigação neste domínio, na última década (Matos, Cruz, \& Almeida, 2011). Segundo a literatura na área de domínio, o desempenho excelente é o resultado de uma multiplicidade de fatores internos e externos interagindo entre si (Gagné, 2007; Serpa, 2016). A investigação (Duda \& Treasure, 2006; Duraud-Rush \& Salmela, 2002) acerca dos atletas com rendimentos excelentes tem demonstrado que certas características, psicológicas e emocionais, estão associadas a estes indivíduos. Vários estudos (Amaral \& Cruz, 2013; Barbosa \& Cruz, 1997; Cruz, 1996; Dias, Cruz, \& Fonseca, 2009) concluíram que os atletas com rendimentos excelentes apresentam níveis elevados de motivação, autoconfiança e concentração, definem objetivos claros e específicos, valorizam o rendimento individual, utilizam a imaginação e a visualização mental, apresentam bom controlo da ansiedade, possuem capacidade para lidar eficazmente com situações imprevistas. Segundo a literatura (Durand-Bush \& Salmela, 2002; Oliver, Hardy, \& Markland, 2010) o perfil psicológico para a excelência no desporto está associado a elevados níveis de motivação, comprometimento, concentração, regulação emocional, competências de autorregulação, resiliência, autoconfiança e autoconceito. Vários autores (Álvarez, Castillo, Duda, \& Balaguer, 2009; Serpa, 2016; Winterstein, 2002) têm procurado compreender o processo de motivação no desporto atribuindo causas a fatores pessoais (e.g., interesses, metas, personalidade) e situacionais (e.g., tarefas atrativas, influências sociais, liderança do técnico). A teoria da autodeterminação defende os constituintes da motivação intrínseca e extrínseca, considerando fatores da personalidade em contexto social, bem como as causas e consequências do comportamento (Deci \& Ryan, 1985). A teoria dos objetivos de realização (Nicholls, 1984) preconiza que o comportamento em contexto de realização é influenciado por fatores pessoais e motivacionais. Segundo Nicholls (1984), as pessoas dirigem a sua motivação com base em dois tipos de orientação, a orientação para a tarefa e a orientação para o ego. A orientação para a tarefa relaciona-se com o êxito no domínio da tarefa, por sua vez, a orientação para o ego pressupõe a demonstração elevada de competência perante os outros (Álvarez et al., 2009). De acordo com Winterstein (2002), a expectativa de êxito está associada a atletas com orientação 
motivacional dirigida para a tarefa, enquanto que, o medo do fracasso se encontra relacionado a atletas com motivação orientada para o ego. A orientação para a tarefa tem sido frequentemente associada a experiências de mestria, e a orientação para o ego com a aprovação social (Saies, Arribas-Galarrag, Cecchini, Luis-De-Cos, \& Otaegi, 2014). Contudo estas definições não têm sido consensuais na literatura. Vários autores (White \& Duda, 1994; Cecchine, González, \& Montero, 2007) têm demonstrado que a orientação para o ego apresenta resultados superiores nos atletas que se encontram em níveis mais elevados de competição e rendimento. A forma como os atletas orientam a sua motivação parece relacionar-se com as disposições individuais e do contexto, onde o treinador assume um papel fundamental tendo em conta a relação de influencia exercida (Serpa, 2016).

A par da motivação, também as emoções têm assumido um papel central na investigação acerca do sucesso desportivo. Sheard e Golby (2009), avaliaram as emoções de jogadores profissionais de rugby em diferentes níveis de competitividade e apuraram que os jogadores de nível intermédio apresentaram valores mais elevados para as emoções positivas quando comparados com os jogadores de nível superior. As respostas emocionais de determinado atleta e a capacidade deste para as regular de modo a poder experienciar emoções adequadas, parece ser uma competência fundamental para o sucesso desportivo (Amaral \& Cruz, 2013). O estudo das emoções associado ao rendimento dos atletas tem vindo a merecer o interesse de vários autores (Cruz, 1996; Cruz \& Barbosa, 1998; Dias et al., 2009; Lazarus, 2000; Matos et al., 2011) afim de se compreender melhor este fenómeno. Tem sido apontado que as emoções positivas neutralizam os efeitos das emoções negativas, proporcionando a adaptação ao stress e auxiliando a aquisição de estratégias de coping (Folkman \& Moskowitz, 2000). As estratégias de coping (e.g., concentração na tarefa, paragem de pensamento, reavaliação positiva) têm assumido um papel relevante $\mathrm{e}$ demonstrado a sua eficácia no sucesso desportivo (Nicholls, Holt, Polman, \& Bloomfield, 2006; Dias et al., 2009). A interpretação e a avaliação das situações influenciam os processos cognitivos, fisiológicos e emocionais, e consequentemente o próprio desempenho (Dias et al., 2009). A capacidade do atleta para compreender as emoções durante e depois da competição, a sua vulnerabilidade a estas emoções e a forma como são geridas, apresentam-se como aspetos fundamentais para o sucesso desportivo (Lazarus, 2000). Para além de fatores de ordem intrapessoal, pesquisas mais recentes têm apontado que a regulação emocional é um processo social e interpessoal (Friesen, Lane, Devonport, Sellars, Stanley, \& Beedie, 2013).

$\mathrm{Na}$ literatura, o desporto tem sido frequentemente associado a fatores motivacionais e emocionais. Considerando-se este quadro conceptual importa ainda conferir relevo ao papel do bem-estar subjetivo, principalmente, no que se relaciona com as emoções positivas e negativas. Segundo Diener (2000), os indivíduos apresentam níveis mais elevados de BES quando experienciam mais emoções positivas e quando desempenham atividades que consideram interessantes e satisfatórias. De acordo com Coleta, Lopes e Coleta (2012) a felicidade correlaciona-se com a satisfação com a vida em geral e com os seus vários aspetos, nomeadamente, as amizades e sentimentos de popularidade. Também em relação às variáveis de cariz demográfico, os autores (Coleta, Lopes, \& Coleta, 2012) apontam a juventude, o sexo feminino e o nível socioeconómico mais elevado, como facilitadoras ou determinantes para o bem-estar subjetivo e felicidade. Num estudo levado a acabo por Jones e Sheffield (2008) junto de jogadores de hóquei e de futebol, os autores avaliaram o efeito do resultado do jogo sobre o bem-estar dos mesmos e apuraram que após uma derrota, os jogadores apresentavam mais ansiedade, raiva e depressão, bem como a presença de sintomas somáticos mais fortes. As emoções sucedem a avaliação efetuada pelo individuo acerca do significado atribuído a cada situação, com implicações positivas e negativas para o bem-estar (Lazarus, 2000). Sempre que é efetuada uma avaliação positiva, em geral, o individuo atinge metas consideraras importantes, experienciando sentimentos de satisfação e afeto positivo (Lazarus, 2000).

Com base nos pressupostos suprarreferidos, o presente estudo pretende apurar a existência de relação entre a orientação motivacional, a regulação emocional e o bem-estar subjetivo, bem como avaliar os níveis de diferenciação em função do rendimento desportivo de jovens atletas. Deste modo, pretende-se contribuir para o enriquecimento do conhecimento científico na área de domínio, bem como para um melhor entendimento dos fatores psicológicos e emocionais, que parecem influenciar o desempenho dos atletas de alta competição.

\section{Método}

\section{Participantes}

A amostra do estudo é constituída por 50 atletas do género masculino com idades compreendidas entre os 14 e os 15 anos, pertencentes aos escalões de formação de um clube de futebol português da primeira liga. Os participantes foram distribuídos por dois grupos de acordo com o rendimento desportivo. Assim, 30 participantes constituíram o grupo A, associado ao escalão sub-15 que apresenta uma mérito desportivo mais elevado, e o grupo B incluiu 20 atletas pertencentes ao escalão sub-14 correspondente a um mérito desportivo menos elevado. $\mathrm{O}$ rendimento foi categorizado mediante a estrutura do sistema de ligas do futebol português onde ambas as equipas atuam. Os elementos do grupo A encontram-se então inseridos no campeonato nacional de juniores, correspondente a um nível de exigência superior, e o grupo B participa nos campeonatos distritais, onde a exigência é inferior quando comparativamente com a anterior.

\section{Materiais}

Para analisar o BES utilizou-se a escala de felicidade subjetiva (Pais-Ribeiro, 2012). Inclui 4 itens e as respostas são dadas numa escala análoga visual com sete posições. A consistência interna da escala foi aceitável 
no presente estudo, apresentando um valor de alpha Cronbach de 0.64 (Marôco, 2011).

A regulação emocional foi medida através da Emotion Regulation Index for Children and Adolescents (ERICA) (MacDermott, Gullone, Allen, King \& Tong, 2010) traduzida e adaptada para a população portuguesa por Reverendo e Machado (2010). É uma medida de autorrelato constituída por 16 itens e permite avaliar a regulação emocional de crianças e adolescentes, dos 9 aos 16 anos de idade. Este instrumento é composto pelas subescalas: controlo emocional, autoconsciência emocional e a responsividade situacional. Neste estudo, a consistência interna mostrou-se favorável com valores de alpha Cronbach que variam entre 0.62 e 0.75 .

O Questionário de Orientação Motivacional no Desporto (QOMD - TEOSQ) é uma versão do Task and Ego Orientation in Sport Questionnaire (TEOSQ), traduzida e adaptada para a língua portuguesa por Fernandes e Serpa (1997) (Massuça, Fragoso, \& Rosado, 2011). Este instrumento permite avaliar a orientação motivacional voltada para a tarefa e/ou para o ego em contexto desportivo. É composto por 13 itens distribuído pelas subescalas, orientação para a tarefa e a orientação para o ego. Neste estudo, a consistência interna revelou-se favorável, apresentando valores de alpha Cronbach que variam entre 0.70 e 0.84 .

\section{Procedimentos}

Para a realização deste estudo foram cumpridos todos os procedimentos éticos necessários, garantindo-se a privacidade e a confidencialidade dos dados. A recolha dos dados concretizou-se junto das camadas jovens (escalões de formação) de uma instituição de futebol portuguesa, na cidade do Porto, a competir na primeira liga de futebol.

Para o tratamento dos dados utilizou-se o Statistical Package for the Social Scienses (versão 22.0). Efetuaram-se análises de estatística descritiva que incluíram medidas de tendência central e medidas de dispersão. Analisou-se a normalidade da distribuição dos dados através do teste de Kolmogorov-Smirnov e Shapiro-Wilk. Recorreu-se a análises de estatística inferencial, nomeadamente, correlações de Pearson, e o teste $t$-Student para comparar as médias dos dois grupos em função das variáveis em estudo. Fixou-se um nível de significância de $0.05(p \leq .05)$ para se considerarem os dados estatisticamente significativos (Marôco, 2011).

\section{Resultados}

Com o objetivo de se avaliar as diferenças nos níveis de felicidade subjetiva dos dois grupos, comparam-se os mesmos em função do seu rendimento desportivo. A leitura da Tabela 1 permite constatar que não se verificam diferenças estatisticamente significativas ( $t=$ $.237, p=.81)$. Assim, a felicidade subjetiva parece não variar em função dos níveis de rendimento dos atletas.
Tabela 1.

Análise das diferenças da felicidade subjetiva em função do rendimento desportivo: Teste $t$-Student

\begin{tabular}{lcc}
\hline Felicidade Subjetiva & $t$ & $p$ \\
\hline Grupo A (alto rendimento) & .24 & .81 \\
Grupo B (baixo rendimento) & & \\
\hline
\end{tabular}

Na Tabela 2 apresentam-se as diferenças nos níveis de regulação emocional do grupo A (rendimento superior) comparativamente com o grupo B (rendimento inferior). Os resultados mostram a existência de diferenças estatisticamente significativas na subescala controlo emocional $(t=2.99, p=.004)$, revelando-se superior no grupo B $(M=79.0, D P=7.92)$. Também na subescala autoconsciência emocional, os resultados indicam diferenças estatisticamente significativas $(t=-2.07, p=$ $.04)$ a favor do grupo B $(M=92.3, D P=9.74)$ quando comparado com o grupo A $(M=86.2, D P=10.49)$. Os resultados indicam que os atletas com rendimento desportivo inferior apresentam níveis de regulação emocional superiores.

Tabela 2.

Diferenças na regulação emocional em função do rendimento desportivo: Teste $t$-Student

\begin{tabular}{lccc}
\hline Regulação Emocional & Rendimento & $t$ & $p$ \\
\hline Controlo emocional & $\begin{array}{l}\text { Grupo A } \\
\text { Grupo B }\end{array}$ & -2.99 & $.00^{* *}$ \\
Autoconsciência emocional & $\begin{array}{l}\text { Grupo A } \\
\text { Grupo B }\end{array}$ & -2.07 & $.04^{*}$ \\
Responsividade situacional & $\begin{array}{l}\text { Grupo A } \\
\text { Grupo B }\end{array}$ & -.39 & .69 \\
\hline
\end{tabular}

A Tabela 3 mostra os resultados das diferenças na orientação motivacional em função do rendimento desportivo. A análise das diferenças entre o grupo A $(M$ $=15.8, D P=3.06)$ e o grupo B $(M=14.0, D P=2.96)$ revelam-se estatisticamente significativas $(t=2.12, p=$ $.04)$. Os resultados sugerem que os atletas com rendimentos superiores, apresentam níveis mais elevados de orientação motivacional para o ego.

Tabela 3.

Diferenças na orientação motivacional em função do rendimento desportivo: Teste $t$-Student

\begin{tabular}{|c|c|c|c|}
\hline Orientação Motivacional & Rendimento & $t$ & $p$ \\
\hline Orientação para a tarefa & $\begin{array}{l}\text { Grupo A } \\
\text { Grupo B }\end{array}$ & -1.60 & .11 \\
\hline Orientação para o ego & $\begin{array}{l}\text { Grupo A } \\
\text { Grupo B }\end{array}$ & 2.12 & $.04 *$ \\
\hline
\end{tabular}

Através da leitura da Tabela 4 verifica-se que a subescala orientação para a tarefa apresenta uma correlação significativa e positiva com a subescala autoconsciência emocional $(r=.48, p=.00)$ e com a subescala responsividade situacional $(r=.59, p=.00)$. Os resultados permitem considerar que a autoconsciência emocional e a responsividade situacional são mais elevadas em atletas com uma orientação voltada para a tarefa. Por sua vez, o controlo emocional, apresenta 
correlação significativa e negativa $(r=-.280, p=.05)$ com a subescala orientação para o ego.

Tabela 4.

Correlações entre a regulação emocional e a orientação motivacional: Correlação de Pearson

\begin{tabular}{lcc}
\hline Regulação Emocional & $\begin{array}{c}\text { Orientação } \\
\text { para a tarefa }\end{array}$ & $\begin{array}{c}\text { Orientação para } \\
\text { o ego }\end{array}$ \\
\hline Controlo emocional & .17 & $-.28^{*}$ \\
Autoconsciência emocional & $.48^{* *}$ & -.17 \\
Responsividade situacional & $.59^{* *}$ & .06 \\
\hline
\end{tabular}

\section{Discussão}

A excelência no desporto tem sido alvo de interesse por parte de vários autores (e.g., Amaral \& Cruz, 2013; Friesen et al., 2013; Matos et al., 2011) que têm procurado compreender este fenómeno através do estudo de variáveis psicológicas de natureza afetiva e emocional, motivacional, de cariz social e do contexto.

Neste estudo, atribuiu-se particular atenção às varáveis de natureza afetiva e emocional, e motivacionais uma vez que, na literatura (e.g., Amaral \& Cruz, 2013; Deci \& Ryan, 1985; Dias et al., 2009; Nicholls, 1984) têm assumido um papel essencial para a compreensão dos fenómenos associados ao alto rendimento. Segundo os resultados do presente estudo, a regulação emocional e a orientação motivacional parecem estar associadas ao rendimento desportivo. Apurou-se que a regulação emocional, especificamente, as dimensões controlo emocional e autoconsciência emocional variam em função do rendimento dos atletas, mostrando resultados superiores nos atletas com um rendimento desportivo inferior. A investigação (e.g., Amaral \& Cruz, 2013; Dias et al., 2009; Lazarus, 2000; Matos et al., 2011) realizada neste domínio tem sugerido que as estratégias de coping têm assumido um papel essencial na gestão das emoções. Assim, estes resultados, em que atletas com rendimentos desportivos mais baixos parecem regular melhor as emoções leva-nos a considerar que estes, possivelmente recorrem com mais frequência a estratégias de coping. Este ajustamento emocional pode dever-se ainda a fatores extrínsecos, tais como a família ou o treinador, uma vez que tem sido apontado na literatura como fatores facilitadores para o desempenho desportivo (Serpa, 2016). A par destes resultados, também a orientação motivacional apresenta correlações significativas com as dimensões da regulação emocional. Mediante estes dados e considerando-se a investigação científica (e.g., Amaral \& Cruz, 2013), o modo como os atletas orientam a sua motivação parece apresentar relação na forma como regulam as suas emoções. Tal como sugerido por vários autores (e.g., Cecchine et al., 2007; White \& Duda, 1994), também nesta investigação se apurou que os atletas com uma orientação motivacional voltada para o ego apresentam níveis superiores de rendimento desportivo. Contudo, estes dados não são consensuais na comunidade científica, havendo autores (e.g., Nicholls, 1984; Winterstein, 2002) que defendem que desempenhos elevados estão associados a uma orientação para a tarefa. No que concerne ao bem-estar subjetivo e considerando-se a literatura (Diener, 2000; Jones \& Sheffield, 2008; Lazarus, 2000), seria expectável que os atletas com rendimentos superiores experimentassem maiores níveis de felicidade, no entanto, este fenómeno não se verificou. Os resultados do presente estudo mostram que os níveis de felicidade não variam em função do rendimento desportivo. Este fato pode dever-se ao envolvimento das emoções, uma vez que, de acordo com Lazarus (2000) as emoções resultam da avaliação acerca do significado atribuído a cada situação, com implicações positivas e negativas para o bem-estar. Com base neste postulado e considerando-se o suprarreferido em relação à regulação emocional, os atletas com uma boa capacidade para gerir as emoções, podem não apresentar implicações significativas ao nível da sua felicidade e do seu bem-estar. A este propósito importa ainda refletir acerca das estratégias de coping e o papel que as mesmas podem desempenhar nas percepções de felicidade. De considerar ainda, a possível influência de outras variáveis nestes resultados, tais como, variáveis de caraterização demográfica e do contexto, que segundo a investigação (Coleta, Lopes, \& Coleta, 2012) têm demonstrado a sua influencia no bem-estar e felicidade.

Destaca-se a importância para o investimento no estudo das estratégias utilizadas para a promoção do desempenho desportivo excelente, de modo a aprofundar o conhecimento sobre a sua eficácia e as implicações destas nos fatores de natureza psicológica. Para tal, importa, potenciar a investigação neste domínio com recurso a metodologias mistas afim de se aprofundar o conhecimento sobre as percepções deste público face ao seu desempenho, às suas necessidades, bem como das estratégias que utilizam. De considerar ainda, a realização de estudos longitudinais com amostras representativas da população, permitindo uma análise do comportamento ao longo do tempo durante as várias fases de competição a que estão sujeitos. Reflete-se ainda acerca da importância para o investimento em programas de promoção de competências psicológicas ajustadas à necessidades deste público, bem como dos outros significativos.

\section{Referencias}

Álvarez, M., Castillo, I., Duda, J., \& Balaguer, I. (2009). Clima motivacional, metas de logro y motivación autodeterminada en futbolistas cadetes. Revista de Psicologia General y Aplicada, 62(1), 35-44.

Amaral, M., \& Cruz, J. (2013). Natureza da percepção de ameaça e preditores das estratégias de regulação emocional na competição: Um estudo com atletas talentosos do futebol. Revista Portuguesa de ciências do desporto, 13(3), 45-59.

Barbosa, L. G., \& Cruz, J. F. (1997). Estudo do stress, da ansiedade e das estratégias de confronto psicológico no andebol de alta competição. Psicologia: Teoria, Investigação e Prática, 2, 523-548.

Cecchini, J.A., González, C., \& Montero, J. (2007). Participación en el deporte y fair play. Psicothema, 19, 57-64. 
Cruz, J. F. (1996). Stress, ansiedade e rendimento na competição desportiva. Braga: Edições do Centro de Estudos em Educação e Psicologia, Universidade do Minho.

Cruz, J. F., \& Barbosa, L. G. (1998). Stress, ansiedade e confronto psicológico na competição desportiva: Uma nova abordagem de natureza cognitiva, motivacional e relacional. Psicologia: Teoria, Investigação e Prática, 3, 21-70.

Coleta, J., Lopes, J., \& Coleta, M. (2012). Felicidade, bem-estar subjetivo e variáveis sociodemográficos, em grupos de estudantes universitários. Psico-USF, 17(1), 129-139.

Deci, E., \& Ryan, R. (1985). Intrinsic Motivation and Self-Determination in Human Behavior. New York: Plenum Press.

Dias, C., Cruz, J., \& Fonseca, A. (2009). Emoções, stress, ansiedade e coping: Estudo qualitativo com atletas de elite. Revista Portuguesa de Ciências do Desporto, 9(1), 9-23.

Diener, E. (2000). Subjective well-being: The science of happiness and a proposal for a national index. American Psychologist, 55, 34-43. doi: 10.1037//0003-066X.55.1.34

Duda, J. L., \& Treasure, D. C. (2006). Toward optimal motivation in sport: Fostering athletes competence and sense of control. In J. Williams (Ed.), Applied sport psychology: Personal growth to peak performance (5th ed.) (pp. 57-82). New York: McGraw-Hill.

Durand-Bush, N., \& Salmela, J. (2002). The development and maintenance of expert athletic performance: Perceptions of world and olympic champions. Journal of Applied Sport Psychology, 14(3), 154-171. doi: 10.1080/10413200290103473

Fernandes, A., \& Serpa, S. (1997). Orientação motivacional em jovens praticantes de hóquei em patins. Lisboa: Instituto Superior de Psicologia Aplicada.

Folkman, S., \& Moskowitz, J. (2000). Positive affect and the other side of coping. American Psychologist, 55, 647-654

Friesen, A., Lane, A., Devonport, T., Sellars, C., Stanley, D., \& Beedie, C. (2013). Emotion in sport: Considering interpersonal regulation strategies. International Review of Sport and Exercise Psychology, 6(1), 139-154. doi: 10.1080/1750984X.2012.742921

Gagné, F. (2007). Ten commandments for academic talent development. Gifted Child Quarterly, 51, 93-118.

Jones, M., \& Sheffield, D. (2008). The impact of game outcome on the well-being of athletes. International Journal of Sport and Exercise Psychology, 5(1), 54-65. doi: 10.1080/1612197

Lazarus, R. (2000). How emotions influence performance in competitive sports. The Sport Psychologist, 14, 229-252.

MacDermott, S. T., Gullone, E., Allen, J. S. King, N. J., \& Tong, B. (2010) The Emotion regulation index for children and adolescents (ERICA): A Psychometric investigation. Journal of Psychology and Behavioral Assessment, 32, 301-314.
Marôco, J. (2011). Análise estatística com o spss statistics ( $5^{\mathrm{a}}$ ed.). Pero Pinheiro: ReportNumber.

Massuça, L., Fragoso, I., \& Rosado, A. (2011). Teste à validade do questionário de orientação motivacional no desporto (QOMD-TEOSQ) em atletas de andebol. Laboratório de psicologia, 9(2), 125-132.

Matos, D., Cruz, J., \& Almeida, L. (2011). Excelência no desporto: Para uma compreensão da "arquitectura" psicológica dos atletas de elite. Motricidade 7(4), 27-41.

Nicholls, J. (1984). Achievement Motivation: Conceptions of ability, subjective experience, task choice, and performance. Psychological Review, 91(3), 328-346. doi: 10.1037/0033-295x.91.3. 328

Nicholls, A., Holt, N., Polman R., \& Bloomfield, J. (2006). Stressors, coping, and coping effectiveness among professional rugby union players. The Sport Psychologist, 20, 314-329.

Oliver, E., Hardy, J., \& Markland, D. (2010). Identifying important practice behaviors for the development of high-level youth athletes: Exploring the perspectives of elite coaches. Psychology of Sport and Exercise, 11, 433-443. doi:10.1016/j.psychsport.2010.05.004

Pais-Ribeiro, L. J. (2012). Validação transcultural da escala de felicidade subjetiva de Lyubomirsky e Lepper. Psicologia, Sáude \& Doenças, 13(2) 157-168.

Reverendo, I., \& Machado, T. (2010). Emotion Regulation Index for Children and Adolescents (ERICA): Tradução e adaptação da versão portuguesa. Faculdade de Psicologia e Ciências da Educação da Universidade de Coimbra, Coimbra, Portugal.

Saies, E., Arribas-Galarrag, S., Cecchini, J. A., Luis-De-Cos, I., \& Otaegi, O. (2014). Diferencias en orientación de meta: Motivación autodeterminada, inteligência emocionl y satisfacción com los resultados desportivos entre piraguistas expertos y novatos. Cadernos de Psicología del Desporte, 14(3), 21-30.

Serpa, S. (2016). Psicologia do desporto. Retirado de http://www.idesporto.pt/ficheiros/file/Manuais/GrauI/ GrauI-02_Psicologia.pdf

Sheard, M., \& Golby, J. (2009). Investigating the rigid persistence paradox in professional rugby union football. International Journal of Sport and Exercise Psychology, 7(1), 101-114.

White, S., \& Duda, J. (1994). The relationship of gender, level of sport involvement, and participation motivation to task and ego orientation. International Journal of Sport Psychology, 25, 4-18.

Winterstein, P. J. (2002). Motivação para a atividade física e para o esporte. In D. De Rose Jr, (Org.) Esporte e atividade física na infância e na adolescência: uma abordagem multidisciplinar (pp. 77-87). Porto Alegre: Artmed. 